\title{
Aerodynamics and Aeroacoustics Investigation of a Low Speed Subsonic Jet
}

\author{
Pedro R. C. Souza \\ Federal University of Uberlândia, Block 5P, Uberlândia, Minas Gerais, 38408-100, Brazil \\ Anderson R. Proença \\ Institute of Sound and Vibration Research, Southampton, Hampshire, SO171BJ, UK \\ Odenir de Almeida \\ Federal University of Uberlândia, Block 5P, Uberlândia, Minas Gerais, 38408-100, Brazil
}

\author{
Rodney H. Self \\ Institute of Sound and Vibration Research, Southampton, Hampshire, SO171BJ, UK
}

(Received 1 June 2016; accepted: 5 October 2016)

\begin{abstract}
Low and high speed subsonic jets have been studied in the last 50 years mainly due to their many applications in industry, such as the discharge of turbojets and turbofan engines. The purpose of this work is to investigate the aerodynamics and the acoustical noise generated by a single stream jet flow operating at low Mach number 0.25 and Reynolds number of $2.1 \times 10^{5}$. The main focus is the flow and acoustical characterization of this low speed jet by applying different experimental techniques for evaluating the velocity field by using measurements with a Pitot tube, hot-wire anemometry, and farfield noise acquisition by free field microphones. In order to verify the validity of aeroacoustic predictions for such a low speed jet, a Computational Fluid Dynamics by means of RANS simulations via k- $\omega$ SST model have been employed coupled with a statistically low-cost Lighthill-Ray-Tracing method in order to numerically predict the acoustic noise spectrum. The sound pressure level as a function of frequency is contructed from the experiments and compared with the noise calculations from the acoustic modeling. The numerical results for the acoustics and flow fields were well compared with the experimental data, thus showing that this low-cost flow-acoustic methodology can be used to predict the acoustic noise of subsonic jet flows, even at low speeds.
\end{abstract}

\section{NOMENCLATURE}

$D_{j}$ Jet's diameter $[\mathrm{m}]$

$U_{j}$ Jet's velocity $[\mathrm{m} / \mathrm{s}]$

$M$ Mach number

$k$ Turbulent kinectic energy [ $\mathrm{J} / \mathrm{kg}]$

$\epsilon$ Turbulent dissipation rate $[\mathrm{J} / \mathrm{kgs}]$

$\theta$ Observer's polar angles [rad]

$R$ Observer's radius [m]

\section{INTRODUCTION}

The noise produced by an aircraft has been an important subject in the past few decades in both industry and academic research. It is well known that noise is generated by different components and by the interaction of external flow and the aircraft parts. According to the aircraft performance, during each phase of flight, one region or piece of equipment should contribute more or less to the "total noise."1 In other words, the aircraft on the ground, while taxing, is on a run-up from the jet exhaust; during the take-off, it is underneath to departure

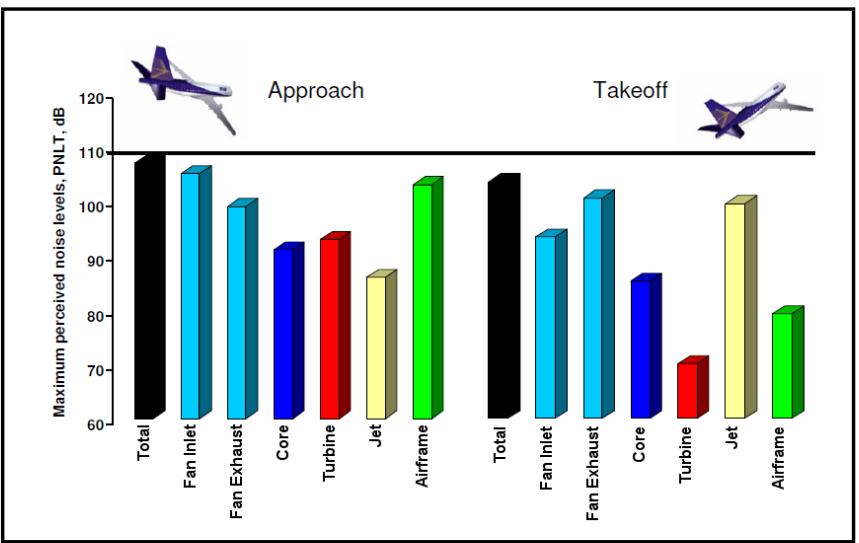

Figure 1. Breakdown of the noise components at take-off and approach. ${ }^{2}$

and arrival paths. If it is over-flying while in route and landing, it produces different noise signatures not only in terms of amplitude but also in its composition, as seen in Fig. 1.

According to Fig. 1, aircrafts have various noise sources, meaning that the engines are one of the major contributors to the total noise. At take-off and climb, the fan exhaust and jet are mainly responsible for the noise levels of an aircraft. During the approach, the engine noise is also considerable. Although high bypass ratio turbofans engines have expe- 
rienced advanced modifications and improvements in the last few years, fan noise and jet noise still play the most important role in terms of noise generation. ${ }^{2}$

Driven by new noise regulations and the need to be an environmental less impactant, the aeronautical industry and academic research centers have invested efforts for understanding and proposing new techniques and ideas to reduce engine and airframe noise. This subject has undoubtedly proven to be quite important in modern aeronautics and is the main motivation for this work.

The different ways to study engine/airframe noise goes from several experimental techniques up to modern numerical models applied for real articles (engines) or scaled prototypes to be tested in the laboratory. Experiments often become prohibited for real scale since the costs involved are too high, leading directly to experiments with reduced model (scaled models) where knowledge about the problem phenomenology, laws of similarity, and practical equipment is really useful.

On the other hand, the numerical approach is split into at least three main branches when considering Computational Fluid Dynamics (CFD):

- Direct Numerical Simulation (DNS) solving all the motion scales of the flow;

- Subgrid Scale (SGS) modeling where LES (Large Eddy Simulation) is one of the examples, solving partially the flow scales;

- Hybrid or RANS (Reynolds Averaged Navier-Stokes) based methods, including the flow and acoustics analogies and empirical models, solving the main characteristics of the flow.

Experimental research of free jets has been reported for at least one century. From the early work of Abramovich ${ }^{3}$ passing by Towsend, ${ }^{4}$ Lilley, ${ }^{5}$ and Lau $\&$ Tester, ${ }^{6}$ among many others, have found that hot-wire Particle Image Velocimetry (PIV) and modern Laser Doppler (LD) applications have an important role in turbulent jet measurements, including the case of jet noise. Measurements made in a low speed air jet (Mach = 0.18 ) with associated cross-spectra and spectral length scales of the axial and lateral velocity components were performed by Harper-Bourne ${ }^{7}$ and enhanced by Morris and Zaman, ${ }^{8}$ thus providing a more complete picture of the relevant turbulent statistics, including a wider range of reference points in the jet through cross spectra and cross correlations, second and fourth order statistics, and comparisons with a RANS prediction method. Non-intrusive techniques have been employed by Mielke et al. ${ }^{9}$ to measure velocity, density, temperature, and turbulence velocity fluctuations in sparsely seeded, high-speed gas flows, used to make measurements in a $25.4 \mathrm{~mm}$ diameter free jet at subsonic and supersonic flow conditions.

Bridges et al. ${ }^{10}$ used PIV to calculate turbulence quantities in nozzle flows from instantaneous 2D velocity maps. Other published works are related to comparison between experimental and numerical results of free turbulent jets. Ghahremanian and Moshfegh ${ }^{11}$ presented numerical results of 3D modeling of an isothermal free jet with four different RANS turbulence models that were validated against hot-wire anemometry data. The comparison showed an excellent agreement between the experimental and numerical results.
Other works in the literature show numerical results that were validated against proper data or results from others, such as Freund ${ }^{12}$ and Stromberg et al., ${ }^{13}$ of a simple round jet flow and acoustics. A more specific analysis, including the use of chevrons, can be seen in the works of Xia et al., ${ }^{14}$ Birch et al., ${ }^{15}$ and Engel ${ }^{16}$ among others.

In this work, a sequential and comprehensive study about the physics of a subsonic free stream jet was proposed by performing controlled experiments for the evaluation of the flow and acoustic fields through the use of a multiprobe Pitot tube, hotwire anemometer, and farfiel acoustic measurements. A complementary numerical analysis was adopted by a hybrid approach based on RANS modeling coupled with a noise prediction method called Lighthill-Ray-Tracing (LRT) - Silva, ${ }^{17}$ for fluid flow calculations and the prediction of the sound sources in the flow and its propagation to an observer in the far-field, respectively.

By considering such a path, the main contribution of this work was to characterize the flow of a low-speed subsonic jet (Mach $=0.25)$ by means of experimental measurements and to use such data to validate a low-cost hybrid RANS-based method coupled with the LRT method for predicting the farfield noise and its directivity by using the fluid flow properties calculated with the RANS technique as input. The experimental data was then used as an original benchmark for the numerical prediction tools, which have constituted a low-cost flowacoustic methodology for being used in industry. The agreement between the numerical solution and experimental data was very good, thus proving that this approach can be used to predict acoustic noise of subsonic jet flows, even at low speeds.

\section{EXPERIMENTAL MEASUREMENTS}

The experimental part of this research was carried out in the Doak Laboratory, which is a Rolls Royce University Technologic Center (UTC) facility located in the Institute of Sound and Vibration Research (ISVR) at University of Souhthampton, United Kingdom. A general description and information about this small scale test facility will be given in the sequence.

The ISVR's Doak laboratory is a $15 \mathrm{~m} \times 7 \mathrm{~m} \times 5 \mathrm{~m}$ anechoic chamber that is fully anechoic down to $400 \mathrm{~Hz}$. The four walls, ceiling, and floor are covered with wedge-type absorbent material. A non-forced exhaust system was composed by a rectangular collector section allowing the air flow to pass through into a small secondary acoustic chamber, as seen in Fig. 2. The air flow was fed from two high pressure compressed air (20 bar) from two storage tanks and the range of velocity available for testing is from Mach 0.2 up to 1 . At these conditions, single jet measurements could be performed on flow regimes characteristic of civil aircrafts. For jet noise measurements, both polar and a transversable azimuthal array of microphones were used to give a complete three-dimensional sound field.

\subsection{Test Article Convergent Nozzle}

The test article used in this work was a $38.1 \mathrm{~mm}$ exitdiameter, convergent, conical nozzle used for most of the tests done at the Doak Laboratory. This nozzle was selected because its aerodynamic and acoustical characteristics were well- 


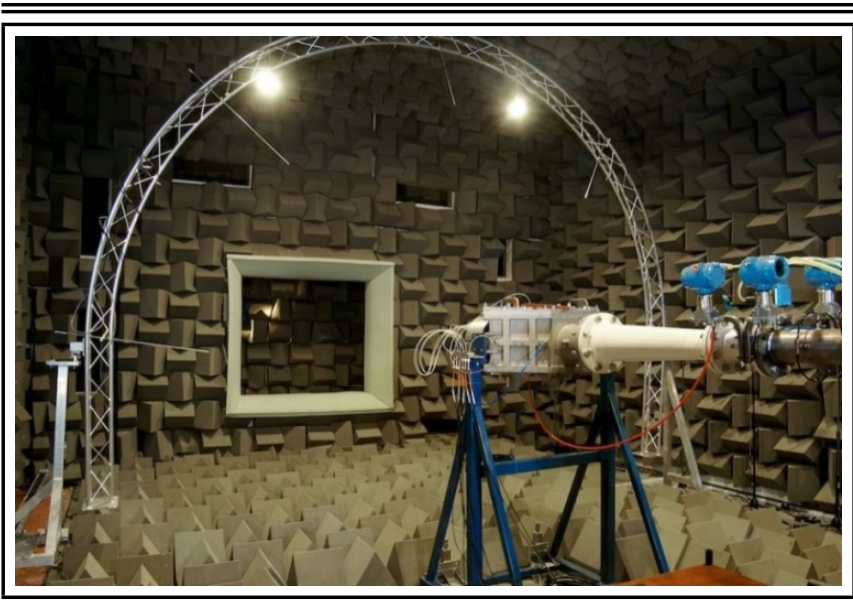

Figure 2. An internal view of the Doak Laboratory ISVR (after Proença ${ }^{19}$ ).

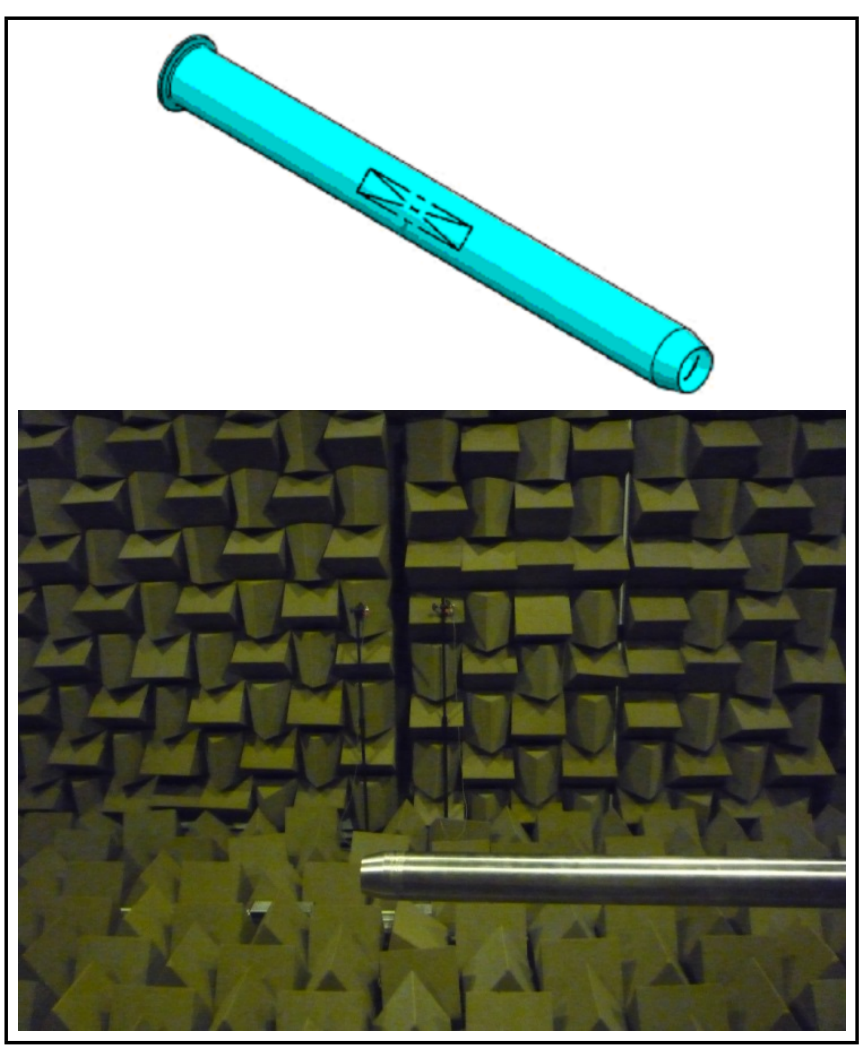

Figure 3. Sketch and picture of $38 \mathrm{~mm}$ diameter (reference nozzle, ISVR).

documented in the Noise Test Facility (NTF) at QinetiQ, ${ }^{18}$ Farnborough, UK.

The subsonic jet was operated from the nozzle at an isothermal condition running at a Mach number of 0.25 . In order to run aerodynamic measurements with a Pitot tube and hot-wire anemometer, a traverse system was placed inside the anechoic room - Fig. 4.

\subsection{Acoustic Noise Measurements}

Acoustical data was acquired by using GRAS Type 40BF microphones, with a frequency range from $10 \mathrm{~Hz}$ to $100 \mathrm{kHz}$ and dynamic range from $40 \mathrm{~dB}$ to $174 \mathrm{~dB}(\mu \mathrm{Pa}),{ }^{20}$ and digitized using a National Instruments NI PCI-4472 acquisition card with a $102.4 \mathrm{kHz}$ sample rate and a 24-bit resolution.

The acoustical measurements were performed only in the far-field. Six different observer angles $40,50^{\circ}, 60^{\circ}, 75^{\circ}, 90^{\circ}$,

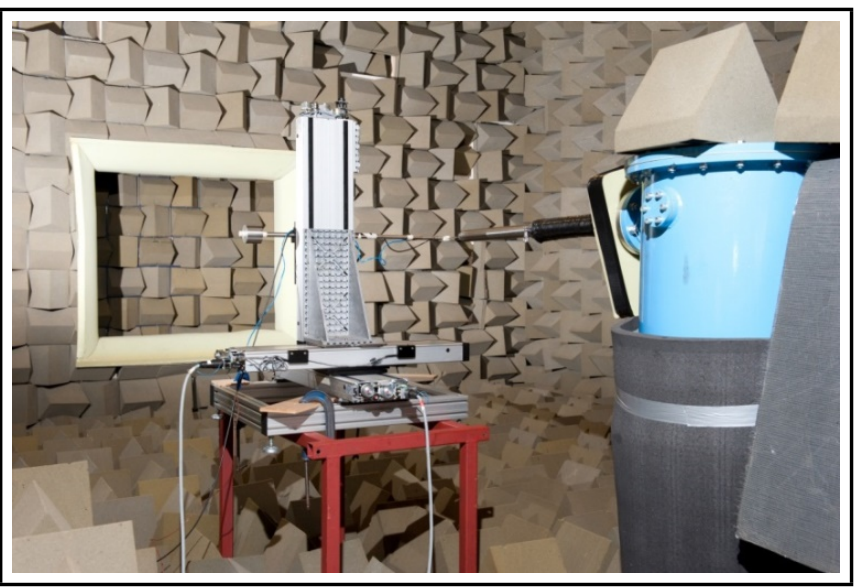

Figure 4. A general view of the Doak Laboratory with a traverse system to hot-wire anemometer and pitot tube measurements — Proença. ${ }^{19}$

and $110^{\circ}$ were applied to acquire the noise signature. Measurements of Overall Sound Pressure Level (OASPL) were achieved by numerically integrating the narrowband spectra with respect to frequency using a trapezium rule method across the entire range of narrowband frequencies. The narrowband data could also be transformed into a one-third octave band spectra by using idealized third-octave filters consistent with ANSI S1.1-1986.

\subsection{Aerodynamic Measurements}

The measurements of the mean flow velocity profiles were performed by using a Pitot tube while the hot-wire anemometer was used for mean flow and turbulence intensity measurements, which is seen in Fig. 5.

The Pitot tube was used to measure the mean flow velocity profiles and the spreading of the jet. Furthermore, it was used as a reference velocity measurement to calibrate the hot-wire sensors.

Hot-wire anemometry was the main measuring system applied in this work. Single hot-wire anemometers are the most common sensors applied in flow measurements because they had a reduced size, their prices were relatively low, they had a high frequency response, and were simple to use. One of the limitations was that it had to be used for low turbulence intensities (up to $10 \%$ ), which was fine for Mach 0.25 free jets. The velocity distributions were acquired along the jet axis to different radial positions and the workspace of mean velocity profile experiments are demonstrated in Fig. 6. The center of the nozzle was located at origin $x, y=(0,0)$, where ' $x$ ' was the jet axis and ' $y$ ' was the radial variation. The red dots symbolize where the data was acquired. Only single hot-wire probes with the points inside the blue rectangle were acquired. Thus, with the Mach number analyzed, 963 points were recorded to the Pitot tube and triple hot-film measurements, whilst 583 to single hot-wire. Additional information for the experimental part of this work can be found in the work of Proena. ${ }^{19}$

\section{NUMERICAL MODELING}

This section is concerned with the mathematical modeling used for the fluid flow and acoustical simulations. The aerodynamic simulations were conducted with the well-known 


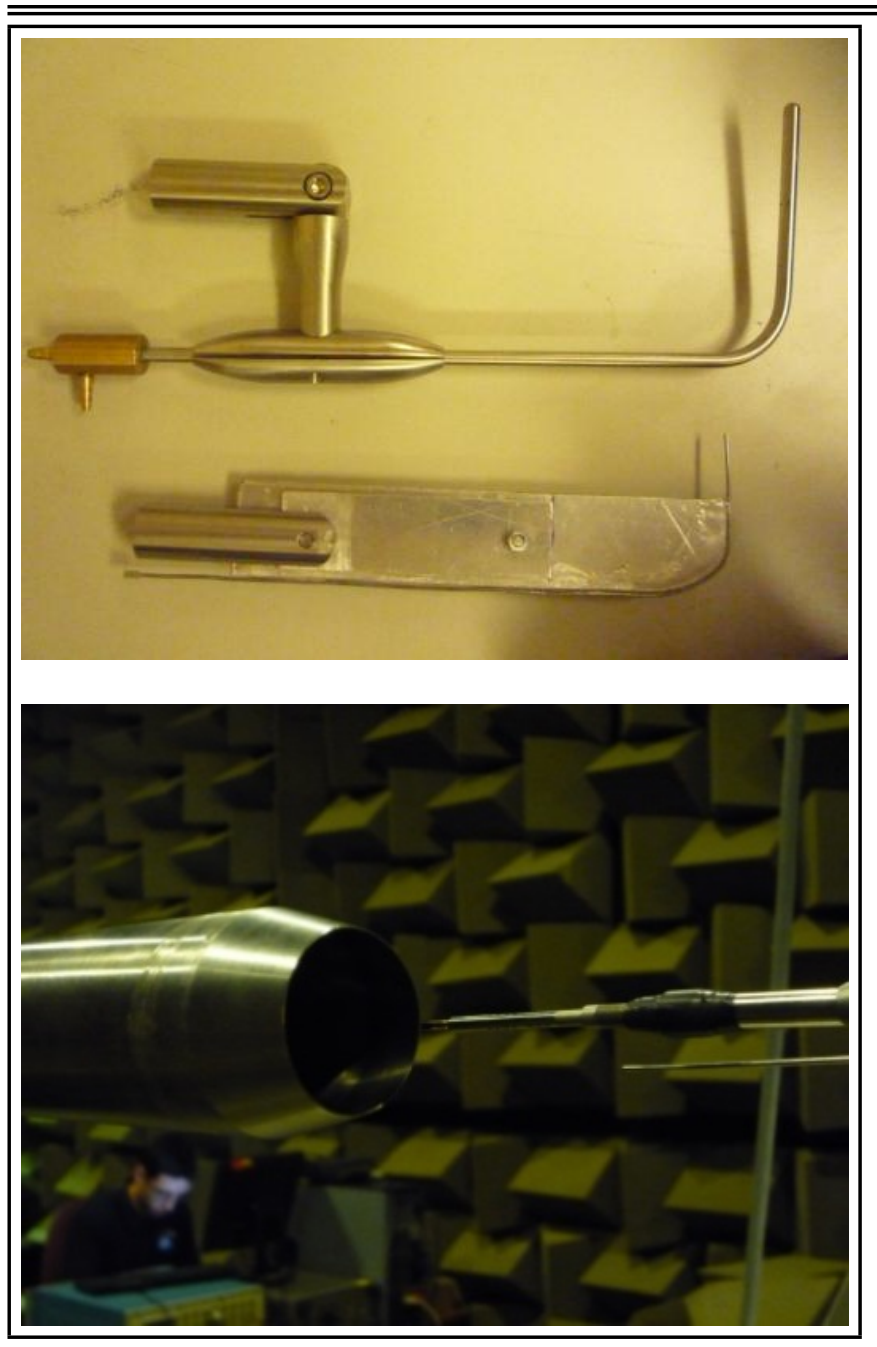

Figure 5. Pitot tubes and single hot-wire sensors — Proença. ${ }^{19}$

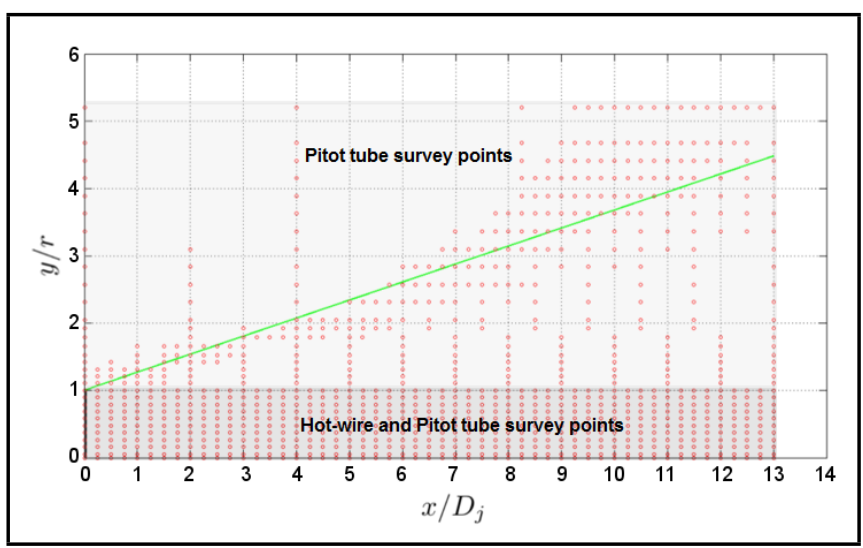

Figure 6. Acquisition points along the region of the jet for aerodynamic measurements — Proença. ${ }^{19}$

CFD++ commercial code ${ }^{20}$ and the acoustical predictions were obtained using the LRT method. ${ }^{17}$

\subsection{Aerodynamics Simulation}

A Reynolds Averaged Navier-Stokes (RANS) approach was used in this work. The compressible steady-state equations of motion were solved in a tridimensional domain.

The equation system that described the problem was composed by the continuity, Navier-Stokes, and energy equations. Upon using RANS, the term $\overline{\rho u_{i}^{\prime \prime} u_{j}^{\prime \prime}}$ that involved the mean of
Table 1. Constants used in the k- $\omega$ SST model.

\begin{tabular}{|c|c|c|c|c|c|}
\hline$\sigma_{k 1}$ & $\sigma_{k 2}$ & $\sigma_{\omega 1}$ & $\sigma_{\omega 2}$ & $\beta_{1}$ & $\beta_{2}$ \\
\hline 0.85 & 1.0 & 0.5 & 0.856 & 0.075 & 0.0828 \\
\hline \hline$\gamma_{1}$ & $\gamma_{2}$ & $\alpha_{1}$ & $\alpha_{2}$ & $\beta^{*}$ & $\kappa$ \\
\hline 0.553 & 0.44 & $5 / 9$ & 0.44 & 0.09 & 0.41 \\
\hline
\end{tabular}

density and velocity fluctuations appeared and the k- $\omega$ SST turbulence model was used for closure. This model solved the transport equations for turbulent kinetic energy $(k)$ and specific turbulence dissipation rate $(\omega)$, by using the equations presented below:

$$
\overline{\rho u_{i}^{\prime \prime} u_{j}^{\prime \prime}}=\frac{2}{3} \delta_{i j} \bar{\rho} k-\mu_{t} \overline{S_{i j}}
$$

where $\mu_{t}$ was the turbulent viscosity (Eq. (2)) and $S_{i j}$ was given by Eq. (3).

$$
\begin{gathered}
\frac{\mu_{t}}{\bar{\rho}}=\nu_{t}=\frac{a_{1} k}{\max \left\{a_{1} \omega, S F_{2}\right\}}, \quad S=\sqrt{\frac{2 \overline{S_{i j} \bar{S}_{i j}}}{\beta^{*}}} ; \\
\overline{S_{i j}}=\left(\frac{\partial \overline{u_{i}}}{\partial x_{j}}+\frac{\partial \overline{u_{j}}}{\partial x_{i}}-\frac{2}{3} \frac{\partial \overline{u_{k}}}{\partial x_{k}} \delta_{i j}\right) .
\end{gathered}
$$

The transport equation for turbulence kinetic energy and the specific turbulence dissipation rate were:

$$
\begin{gathered}
\frac{\partial(\bar{\rho} k)}{\partial t}+\frac{\partial}{\partial x_{j}}\left(\bar{\rho} \tilde{u}_{j} k\right)=\frac{\partial}{\partial x_{j}}\left[\left(\mu+\sigma_{k} \mu_{t}\right) \frac{\partial k}{\partial x_{j}}\right]+\tilde{P}_{k}-\beta^{*} \bar{\rho} k \omega \\
\frac{\partial(\bar{\rho} \omega)}{\partial t}+\frac{\partial}{\partial x_{j}}\left(\bar{\rho} \tilde{u}_{j} \omega\right)= \\
\frac{\partial}{\partial x_{j}}\left[\left(\mu+\sigma_{k} \mu_{t}\right) \frac{\partial \omega}{\partial x_{j}}\right]+\frac{\gamma}{\nu_{t}} P_{k}-\beta \bar{\rho} \omega^{2} \\
+2\left(1-F_{1}\right) \bar{\rho} \sigma_{\omega 2} \frac{1}{\omega} \frac{\partial k}{\partial x_{j}} \frac{\partial \omega}{\partial x_{j}}
\end{gathered}
$$

More details about functions such as $P_{k}, \tilde{P}_{k}, F_{1}, F_{2}$, and $C D_{k \omega}$ can be found in Menter's ${ }^{21}$ work. This model, like any other, brought a large number of empirical constants. Except for constants such as $\beta^{*}$ and $\kappa$, all the others had to obey Eq.6; the values used for them in this study are listed in Table 1.

$$
\phi=\phi_{1} F_{1}+\phi_{2}\left(1-F_{1}\right)
$$

where $\phi$ is a constant.

Several tests were made previously in order to find the best turbulence model for this problem, although these tests will not be shown here because they are not the focus of this article.

The governing equations closed with the k- $\omega$ SST model were solved with a second order accuracy through a Finite Volume formulation. As the jet flow was at Mach lower then 0.3, a preconditioning approach was necessary to stabilize the solution. The final result was obtained when the residual dropped 5 orders of magnitude.

\subsection{Aeroacoustics Prediction}

The sound pressure levels generated by the jet were calculated by the Lighthill Ray-Tracing method (LRT), 17 by using the mean flow field characteristics previously calculated by the 


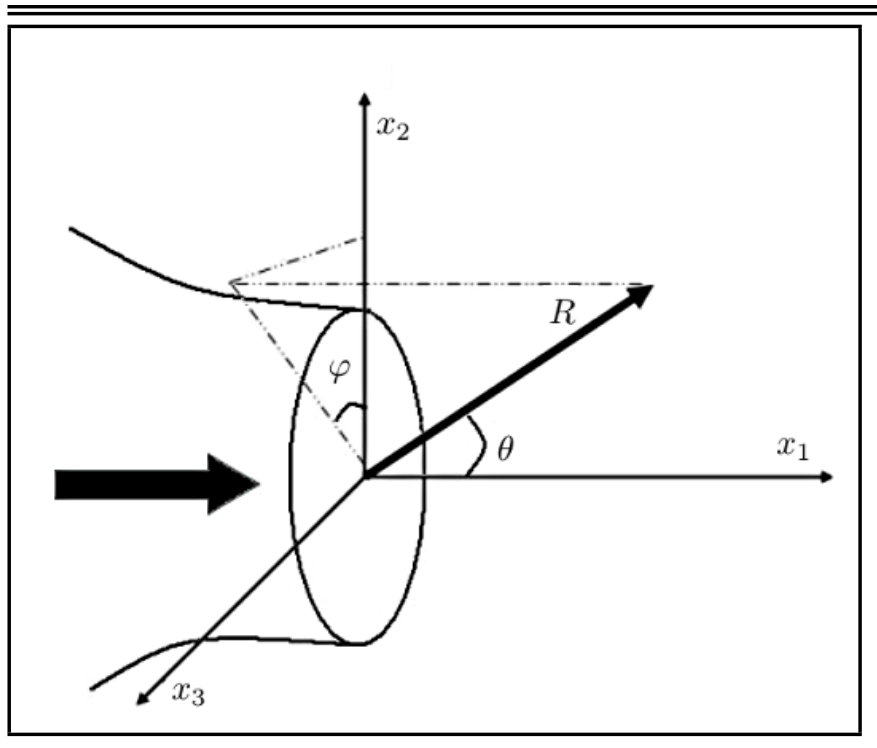

Figure 7. The coordinate system used for the integrations. ${ }^{19}$

CFD code. An external Fortran code was implemented in order to receive the CFD input data as $u, c, T, \rho, k, \omega$ and to compute the acoustical noise by discretizing the jet by virtual sound sources further propagated to the farfield by the RayTracing method.

In fact, this method required the use of the standard Lighthill equations for noise calculations coupled to the Ray-Tracing methodology $\mathrm{y}^{22}$ in order to account for the refractions of sound waves due to velocity gradients that were present in the flow field. Thus, this approach resulted in a better modeling of the sound propagations than other well-known RANS-based acoustic methods like the MGBK. ${ }^{23}$

According to Jordan and Gervais, ${ }^{24}$ the acoustic field generated by a jet flow is:

$$
p(\bar{y}, \theta)=A I_{i j k l} \operatorname{dir}(i j k l)
$$

where $A$ is given by Eq.(8), the fourth-order autocorrelation function for a unit volume of turbulence $I_{i j k l}$ and the source directional patterns $\operatorname{dir}(i j k l)$ can be calculated by Eqs (9) and (10).

$$
\begin{gathered}
A=\frac{\bar{\rho}}{16 \pi^{2} c^{2} R^{2}\left[1-M_{c} \cos (\theta)\right]^{5}} \\
I_{i j k l}=\int \frac{\partial^{4}\left(\overline{v_{i} v_{j} v_{k} v_{l}}\right)}{\partial \tau^{4}} d^{3} \bar{r} \\
\operatorname{dir}(i j k l)=\frac{1}{2 \pi} \int_{0}^{2 \pi}\left(\frac{x_{i} x_{j} x_{k} x_{l}}{x^{4}}\right) d \varphi .
\end{gathered}
$$

Using the coordinate system from Fig. 7 and integrating Eqs. (9) and (10) it, it possible to obtain Eqs. (11) and (12):

$$
\begin{aligned}
& p(\bar{y}, \theta)= \\
& A\left(\cos ^{4} \theta\right) I_{1111}+\frac{6}{8} A\left(\sin ^{4} \theta\right) C_{1} I_{1111} \\
& +2 A\left(\cos ^{2} \theta \sin ^{2} \theta\right) C_{2} I_{1111}+4 A\left(\sin ^{4} \theta\right) C_{3} I_{1111} \\
& +4 A\left(\cos ^{2} \theta \sin ^{2} \theta\right) C_{4} I_{1111}+2 A\left(\sin ^{4} \theta\right) C_{5} I_{1111}
\end{aligned}
$$

$$
\begin{aligned}
I_{1111}(\Omega)= & {\left[\frac{\sqrt{\pi}}{4} \frac{c_{l}^{5}}{\alpha_{T}^{3}} \Delta^{2}\left(\frac{3}{2}-\beta\right)^{-\frac{13}{2}}\right] } \\
& *\left[\rho^{2} \tau_{0}^{4} \Omega^{4} k^{7 / 2} \exp \left(-\frac{\tau_{0}^{2} \Omega^{2}}{8}\right)\right] .
\end{aligned}
$$

More details about this method's functions such as the modified convection factor $(\Omega)$ and the convective Mach number $\left(M_{c}\right)$, along with all its constants are available in previous works of Engel ${ }^{16}$ and Silva. ${ }^{17}$ In this fourth-order correlation function (Eq. (12)), there was one calibration coefficient $(\alpha T)$ which needed to be defined based on the experimental data.

Now, the acoustic field generated by the jet could be calculated, however, Eqs. (11) and (12) do not account for any refraction effects caused by the velocity gradients in the flow. In order to incorporate this into the model, Silva ${ }^{17}$ used the standard acoustic ray propagation equations from Pierce ${ }^{22}$ and Blokhintzev's ${ }^{25}$ invariant definition to get to Eq.(13). This equation can calculate the sound pressure level variation due to the refraction effects.

$$
\begin{aligned}
& \Delta S P L= \\
& 10 \log _{10}\left[\frac{B_{\text {source }}}{B_{\text {farfield }}}\left[\frac{N_{\text {rayw/ref }}}{N_{\text {rayw/oref }}}\right]_{\text {farfield }}\right] ; \\
& B=\frac{\left|v_{\text {ray }}\right|}{\left(1-\bar{u}_{i} s_{i}\right) \bar{\rho} \bar{c}^{2}} .
\end{aligned}
$$

Equation (13) evaluates the terms $V_{\text {ray }}$ and $s_{i}$ calculated from ray propagation equation, in both the sound source and farfield locations. It also counts the number of rays $N_{\text {ray }}$ that cross the same area in farfield with and without the refraction effect.

By calculating this $\Delta$ and summing the result with the sound pressure levels obtained from Eq. (11), the LRT method can calculate the SPL from a jet accounting for its refraction effect.

\subsection{Geometry and Computational Mesh}

The tridimensional domain was built with a block structured hexahedral mesh, refined in the nozzle's exit and along the jet's mixing layer (Fig. 8). The computational domain is $80 D_{j}$ long and $40 D_{j}$ wide from the jet's exit. The nozzle penetrates $1 D_{j}$ in the domain and has a $8 D_{j}$ length tube behind it. This setup was for reproducing closely the experimental conditions and maintaining the boundary condition as far as possible from the region of interest. Various grid independency tests were made in order to get to the 2 million elements mesh used in this work. These results are not shown here for brevity.

\subsection{Boundary Conditions and Setup}

The conditions used by the simulations were the same measured in the experiments. The fluid is was air, the ambient pressure and temperature were $101871 \mathrm{~Pa}$ and $291.23 \mathrm{~K}$, respectively, and the mean velocity measured at the nozzle's exit was $85 \mathrm{~m} / \mathrm{s}$. The boundary conditions were a viscous wall function for the jet's nozzle and tube, stagnation pressure, and temperature set to $106397.931 \mathrm{~Pa}$ and $294.870 \mathrm{~K}$ at the jet's inlet, simple back pressure imposition at outflow and Riemann invariants for the fairfield and domains inflow. 


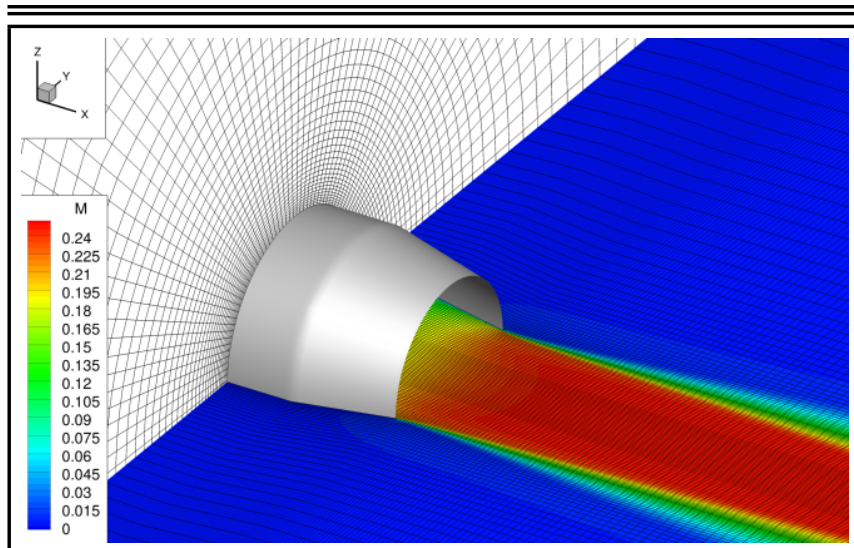

(a) Mesh refinement of the nozzle.

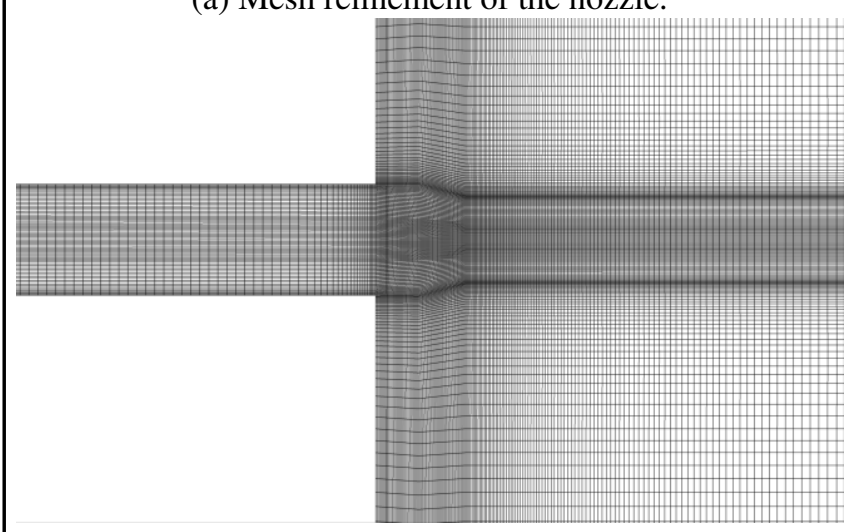

(b) Mesh detail in the nozzle region.

Figure 8. Computational domain and mesh detail. Computational domain and mesh detail.

\subsection{Noise Prediction Parameters}

The noise radiated from the jet encounters a quiescent flow in the farfield with pressure and temperature equaled to the ambient. To run the ray tracing methodology, sound sources needed to be placed into the flow field; as expected, the position of these sources produced great effects in the final acoustical results. When using the ray tracing method in different problems, it is recommended to place the sources in each mesh node in order to keep the precision from the earlier calculations. However, this was unnecessary for a jet flow, as it is known from theory and experiments that the noise produced by this kind of flow is originated mostly in the jet's mixing layer. In fact, the noise generated by a jet flow originates from high turbulent kinetic energy zones spreading around the potential core from 0 up to 6 nozzle diameters in the streamwise direction.

By tracking an iso-surface of low turbulent kinetic energy it is possible to delimitate a tridimensional region where the sound generation is important. Therefore, if sound sources were distributed inside this region, no errors will be carried in the further calculations. In this work, several tests were made with the source number and positions, thus leading to the use of a tridimensional region delimited by $10 \%$ of the jet's maximum turbulent kinetic energy and $10^{3}$ sound sources distributed inside it.

\section{RESULTS}

The results are split in two sub-sections, being firstly shown the aerodynamics characterization of the fluid flow by means

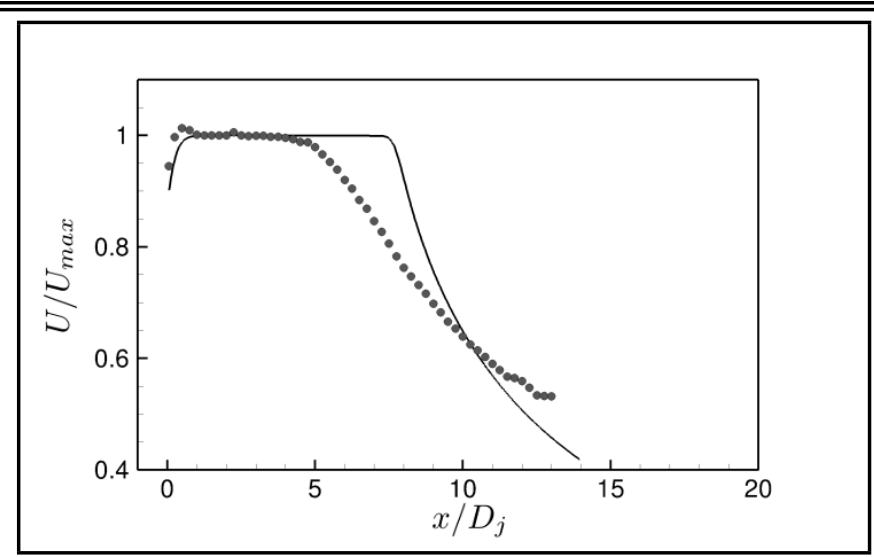

Figure 9. The velocity distribution along the jet axis. - CFD; $\bullet$ Experiments (hot-wire).

of velocity profiles acquired by the Pitot tube and the single hot-wire anemometer. These results are seen in terms of the jet's centerline velocity, radial velocity profiles and the jet's wake characterization contours. The numerical results were compared against the experimental ones in order to validate the flow simulation by employing the k- $\omega$ SST model. The acoustic noise from the experimental measurements and numerical results were presented in sequence by showing the sound pressure level spectra for different observer angles and the overall sound pressure level graph. The acoustic simulations were made aiming to validate the LRT method to describe the physics of the sound generation and propagation of this low-speed subsonic jet. $U / U_{\max } \quad x / D_{j}$

\subsection{Aerodynamics Results}

Results from hot-wire measurements for the normalized mean velocity from the jet's centerline, distributed along the jet axis domain, from 0 up to 13 diameters, at Mach number 0.25 and Reynolds number of $2.1 \times 10^{5}$ (based on the jet diameter and the jet velocity $U_{j}$ ), is shown in Fig. 9. The velocity was expected to be at a maximum and unchangeable until 4 to 5 diameters from the exit nozzle along the centerline, defining the potential core length - Abramovich. ${ }^{3}$ As can be seen from Fig. 9, the potential core ends between 4 to 4.5 diameters downstream from the jet axis.

On the other hand, the numerical model applied in the RANS calculation over predicts the length of the core region, mainly due to the vortex-stretching and turbulence heterogeneity of the 3D flow-field not completely captured with RANS modeling. Although this trend was already expected by the RANS calculations for axisymmetric jets, some turbulence models could enhance the prediction. In general, the length of the potential core was over-predicted by numerical simulations in the range of 6 and 10 diameters, especially when considering standard $\mathrm{k}-\epsilon$ turbulence modeling. ${ }^{26,27}$ In addition to the wrong location of the potential core in Fig. 9, the rate of decay of the centreline axial velocity was also not fairly well approximated. Despite this well-known limitation of Reynolds average simulations, the results showed a reasonable agreement to the experimental data when observing other fluid flow data.

In order to check the trend and the accuracy of the potential core lengths prediction showed in Fig. 9, the axial velocity centerline distribution was obtained with a hot-wire and Pitot tube. Additionally, the centerline for Mach condition $M=0.25$ was 


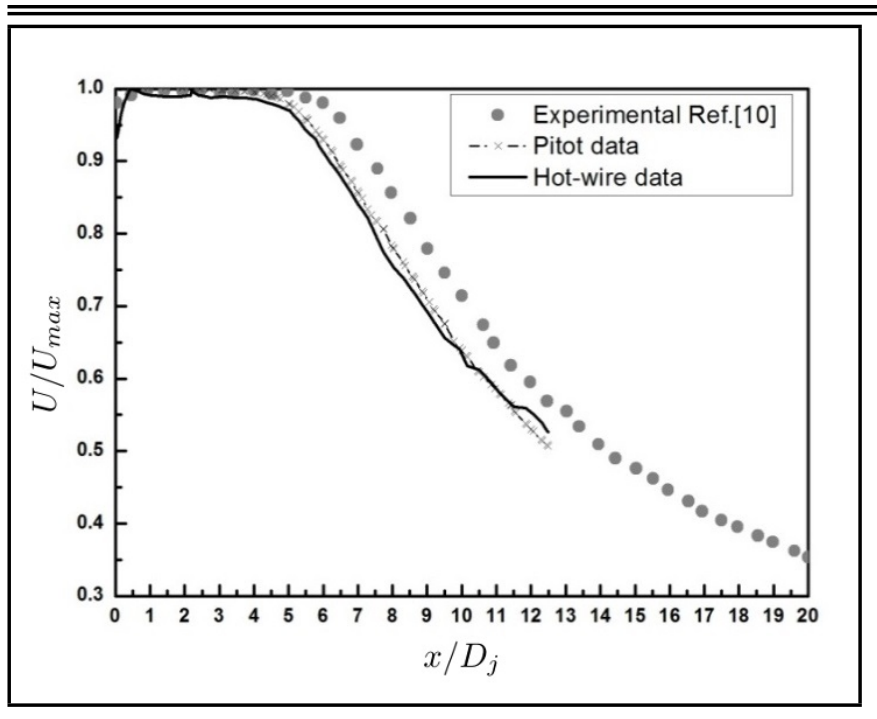

Figure 10. The velocity distribution along the jet axis for the centerline at Mach 0.25. - Hot-wire; $-\times-$ Pitot tube; • Morris and Zaman. ${ }^{8}$

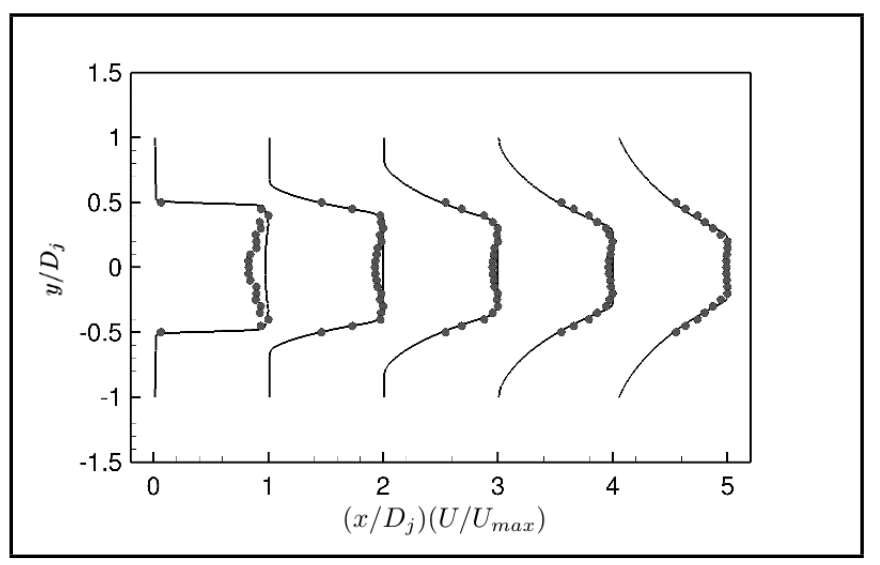

Figure 11. The velocity distribution along the jet radius. - CFD; $\bullet$ Experiments (hot-wire).

also compared to other experimental references from Morris and Zaman. ${ }^{8}$ These comparisons can be seen in Fig. 10. It can also be seen that at Mach number 0.25 the axial velocity profiles from the Pitot and the hot-wire system are very similar. Far away around eleven diameters from the exit nozzle, the hot-wire results showed some inconsistencies that could be related to some unknown flow variation imposed by the compressor system during the measurement. The work of Morris and Zaman ${ }^{8}$ shows only the results for Mach 0.25 , alleging the fragility of the modern hot-wires probes with a high frequency response to high and unsteady flows. Even though a slight difference was seen in the size of the potential core (approximately 6 diameters in Morris and Zaman ${ }^{8}$ ), the trend among the curves are consistent and show a similar rate decay. As stated before, the measurements performed in this work for the length of the potential core are within the experimental range cited in classical literature.

Despite a difference between the numerical and experimental results for the velocity distribution along the jet axis, a better agreement was shown for the radial velocity according to Fig. 10. Here, the experimental data was mirrored by the centerline in order to well characterize the jet's wake at streamwise locations of $x / D=0.0,1.0,2.0,3.0,4.0$ and 5.0. Inside the potential core region, less than 5 nozzle diameters, the numer-

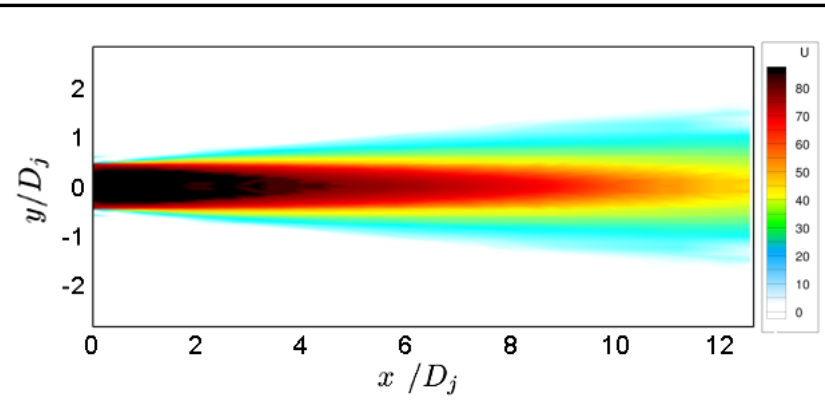

(a) Experimental field

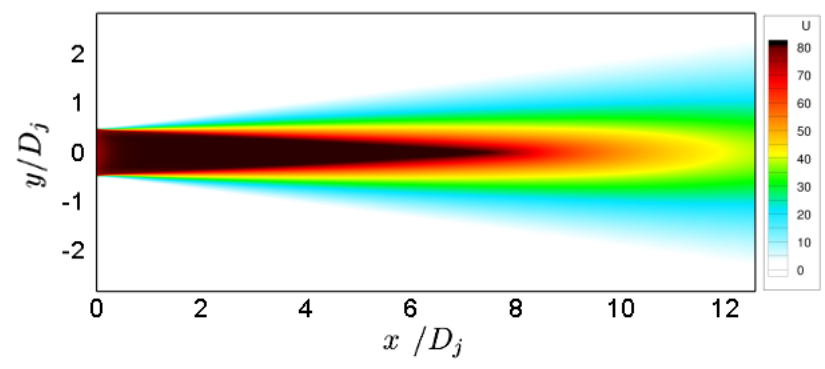

(b) RANS simulation

Figure 12. The jet's wake velocity contours with the Pitot tube data and CFD simulation at Mach 0.25.

ical results fit well against the experimental data. For $x / D=$ 0.0 and 1.0, some discrepancies were be noticed, essentially related to the predictions having smother profiles due to the boundary conditions imposed to the flow.

To explore the flow field of this single jet, a wake flow characterization was considered by its velocity distribution (contours). Since the whole experiment covered 963 points from Pitot tube and 593 points for hot-wire probes, it was possible to create the mean velocity contours. Thus, all lines measured were considered and, such as in the previous sub-section, the data was mirrored along the jet centerline to better characterize the wake. Although it seemed to be merely illustrative or qualitative, it helped to summarize the information given from the velocity profiles. Figure 12 (a) shows the experimental mean velocity distribution from the exit nozzle to thirteen diameters downstream the jet axis, also covering two diameters from the center of the nozzle. In Fig. 12 (b), the numerical results are presented, where it is possible to see the potential core and the shear layer pattern. As mentioned before, the RANS simulation was not able to predict the length of the potential core and a slight difference was also shown in the shear layer spreading. These discrepancies are important to be considered and are expected to have some influence in the noise predictions since the LRT model was based on the primitive variables $(u, v, w)$ and turbulence quantities $(k, \omega)$ of the fluid flow. Figure 12 shows Jet's wake velocity contours with Pitot tube data and CFD simulation, for Mach 0.25 .

Figure 13 shows the jet's wake velocity contours obtained with hot-wire data. As stated earlier, the survey points region for the hot-wire sensor was smaller, with only 593 points inside the potential core region. Thus, the shear layer was not entirely seen, as shown in Figure 12 (a). However, the results were consistent and allowed a good description of the jet's wake.

Centerline variation of axial turbulence intensity is given in Fig. 14. It was known that for an application of a single 


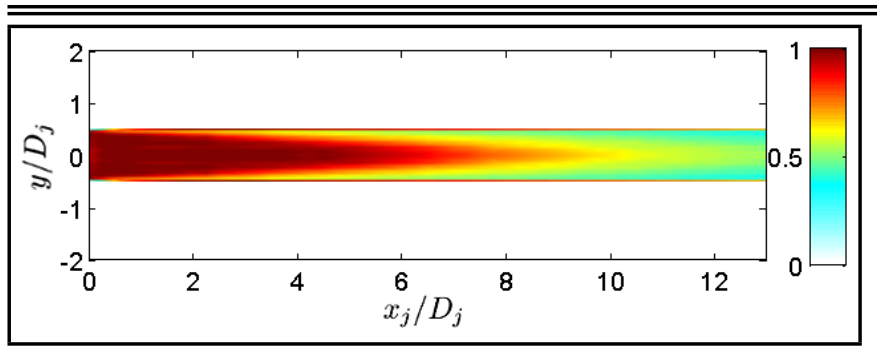

Figure 13. The jet's wake velocity contours with the hot-wire data at Mach 0.25 .

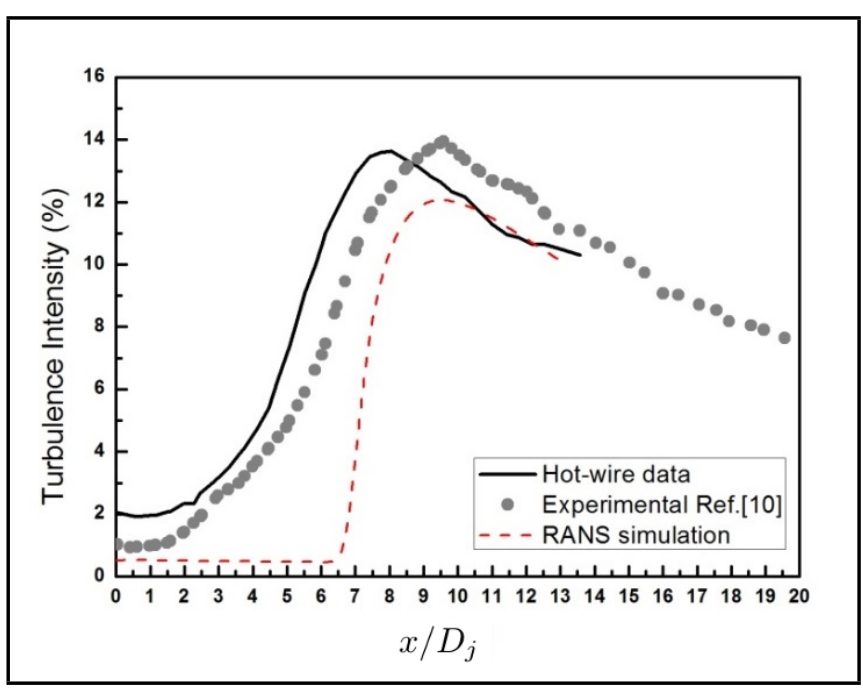

Figure 14. The turbulence intensity along the jet axis for centerline at Mach 0.25. - Hot-wire; • Morris and Zaman, ${ }^{8}$ - - - RANS simulation.

wire for measuring fluctuations, a reliable range to the sensor needed to be around $10 \%$ to $15 \%$ of the mean value. In this case, it can be seen that the peak turbulence intensity reaches approximately $14 \%$ at $x / D_{j}=9.5$ downstream. This result was consistent with other experimental measurements available, such as the work of Morris and Zaman. ${ }^{8}$ It was also known that along the shear layer of a subsonic jet, the maximum value of turbulence intensity was around 15\%. In Fig. 14 it is clear that the RANS calculation under-predicts the peak location and intensity. It was important to emphasize that the turbulence model coefficients have not been optimized for the studied case, assuming the standard values as seen in Table 1.

\subsection{Aeroacoustics Results}

The fluid flow predictions obtained with the RANS simulations were used as input information for the LRT method. The results of the sound pressure spectra are presented for observer angles varying from $40^{\circ}$ to $110^{\circ}$, as shown in Fig. 15 . During the acoustical measurements, the arc of microphones was positioned at approximately $100 D_{j}$ from the nozzle exit and the noise data were corrected for a lossless condition at $1 \mathrm{~m}$. The results were presented in terms of $1 / 3$-octave band spectra. Figure 15 shows the comparison between the experimental and numerical data for all angles proposed. According to the fluid flow and acoustics, the sound sources are convected downstream by the mean flow and the maximum noise is radiated and expected to happen at observer angles between $30^{\circ}$ and $50^{\circ}$, thus making its directivity more pronounced.

By inspecting the sound pressure spectra in Fig. 15, the most significant difference between the numerical predictions and experimental data was found for a $40^{\circ}$ angle. Even though the RANS simulations showed discrepancies for the fluid flow, it was possible to affirm that the LRT method was capable to predict the sound spectra with an acceptable level of agreement for all the angles investigated. However, at smaller (shallow) angles, such as $40^{\circ}$ and $50^{\circ}$, the LRT started to lose its precision since there was a very large noise attenuation (zone of silence) due to the refraction effects caused by the fluid flow these effects have been shown in the literature ${ }^{2,17,28}$ among others. Despite such discrepancies at this angle, it was important to state that this difference was not more than $2 \mathrm{~dB}$.

In Fig. 15(e), the peak noise was predicted fairly well by the LRT method, despite the fact that the peak noise was strongly related to the end of the potential core. As seen before, there was a mismatch between the fluid flow prediction and the experimental data for the length of the potential core. In this case, such discrepancy was not considerable to change the peak noise level.

Finally, Fig. 16 shows the overall sound pressure level (OASPL) by comparing the LRT results with the experimental data. It was possible to see that the LRT method could be used to quantify the acoustical noise of single stream jet flows with reasonable accuracy and relatively low computational cost. In terms of overall SPL, the difference of $2 \mathrm{~dB}$, as stated before, at $40^{\circ}$ is better noticed in Fig. 16.

\section{CONCLUSIONS}

This paper has described aerodynamics and aeroacoustics characterization of a low-speed subsonic jet operating at Mach 0.25 through experimental and numerical techniques. The main goal was to couple experimental and numerical tools to fundamentally study this kind of flow pattern. The experimental data gathered with the Pitot tube and hot-wire anemometer was used as an original benchmark for the validation of a RANS-based method coupled with an acoustic noise modeling (LRT method) to predict the noise at specific locations in the farfield, providing a relatively low-cost methodology that could be applied in the aeronautical context. The results were discussed sequentially from the aerodynamics experimental and numerical data, which compounds most of the efforts in terms of experiments, post-processing and analyses. Additionally, some experimental results are also compared with other data available from literature identifying some possible drawbacks in such kind of measurements. Deviations were observed in the fluid flow predictions and are purely related to the RANS approach considered. The acoustic results are shown to six observer positions in the farfield. The experimental data is confronted to the numerical RANS-based method coupled with the LRT method, thus showing a very good agreement even for shallow angles. The numerical results for the acoustic and flow fields were well compared with the experimental data, showing that this low-cost flow-acoustic methodology can be used to predict acoustic noise of subsonic jet flows, even at low speeds.

\section{ACKNOWLEDGMENTS}

The study reported herein was partially funded by EMBRAER and FAPESP under the GRANT N ${ }^{\circ} 06 / 52568-7$ The 


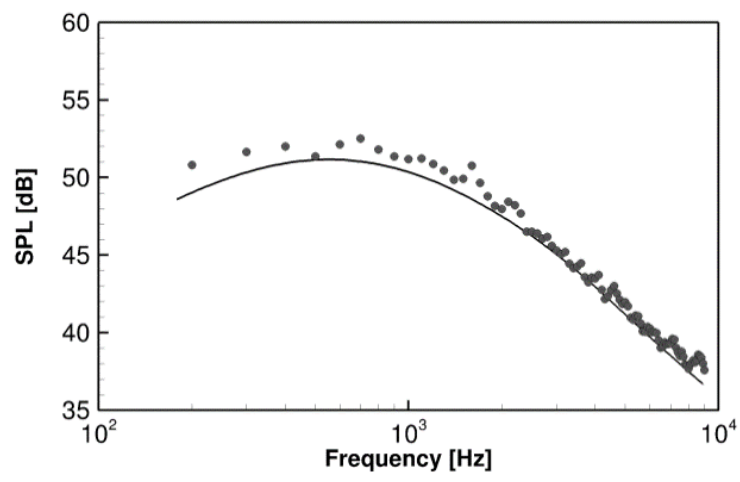

(a) $40^{\circ}$ angle

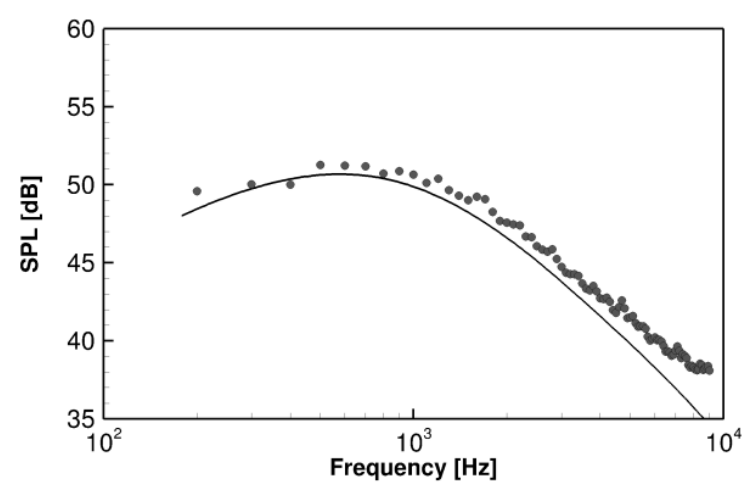

(c) $60^{\circ}$ angle

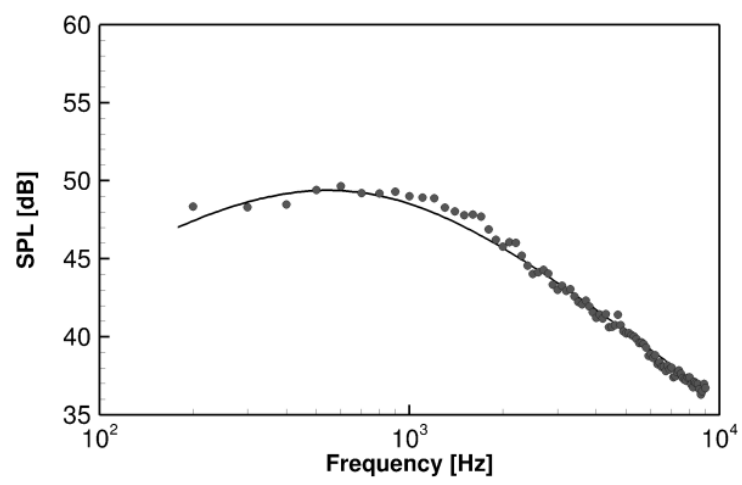

(e) $90^{\circ}$ angle

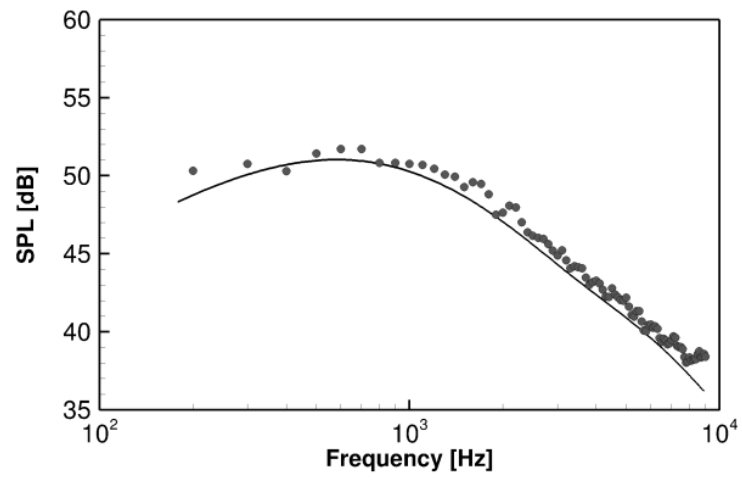

(b) $50^{\circ}$ angle

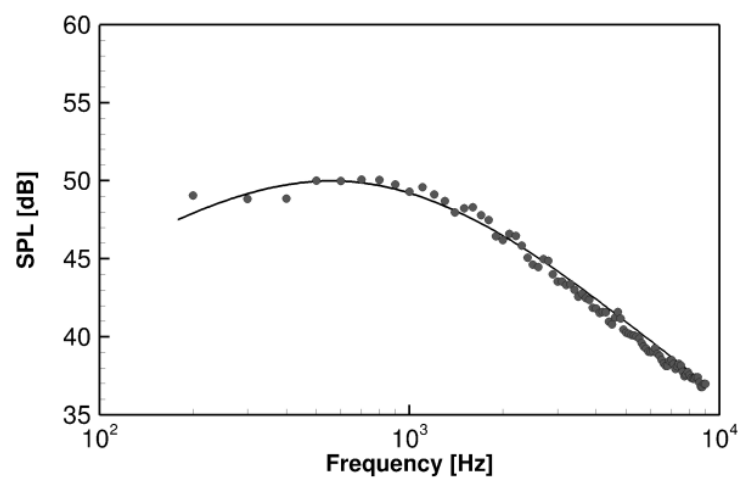

(d) $75^{\circ}$ angle

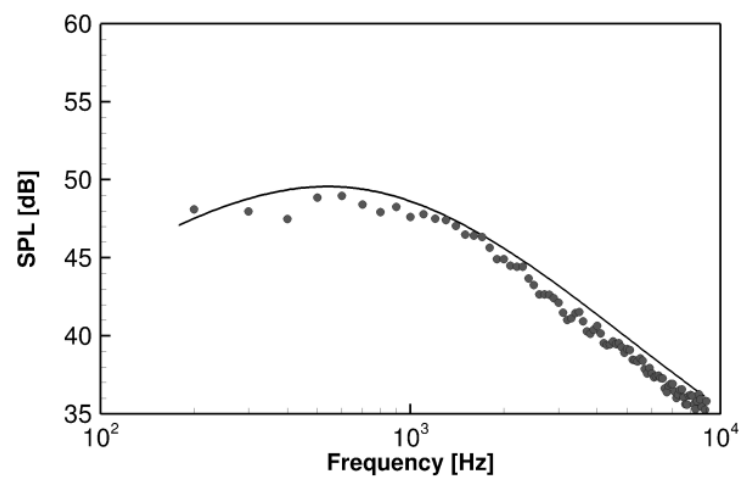

(f) $110^{\circ}$ angle

Figure 15. The sound Pressure Level Spectra at Mach number 0.25 single jet for angles varying from $40^{\circ}$ up to $110^{\circ}$. — LRT; $\bullet$ Experiments.

authors also would like to thank the financial support provided by Coordenação de Aperfeiçoamento de Pessoal de Ensino Superior (CAPES) and the Fundação de Amparo à Pesquisa do Estado de Minas Gerais (FAPEMIG). The first author gratefully acknowledges the support received at the Institute of Sound and Vibration for the conclusion of his MS thesis. The second author also thanks the collaboration between the Institute of Sound and Vibration Research (ISVR) - United Kingdom and the Faculty of Mechanical Engineering at Federal University of Uberlândia (UFU) — Brazil.

\section{REFERENCES}

1 Smith, M. Aircraft noise. Cambridge aerospace series, Cambridge University Press, Cambridge, New York, (1989), Vol. 3. http://dx.doi.org/10.1017/cbo9780511584527
2 Almeida, O. Aeroacoustics of dual-stream jets with application to turbofan engines, - $\mathrm{PhD}$ diss., Aeronautics Institute of Technology, So Jos dos Campos, (2009). http://dx.doi.org/10.13140/RG.2.2.30128.56326

3 Abramovich, G. N. The theory of turbulent jets, Ed. Schindel, L.H., The MIT Press Classics, (1963).

4 Townsend, A. A. The structure of turbulent shear flow, Cambridge University, (1956). http://dx.doi.org/10.1002/zamm.19760560921

5 Lilley, G. M. On the noise from air jets, Aeronautical Research Council, London, England, (1948).

${ }^{6}$ Lau, J. C. and Tester, B. J. An evaluation of the lighthill analogy for jet mixing noise generation: Using LV turbulence, source location and spectral noise 


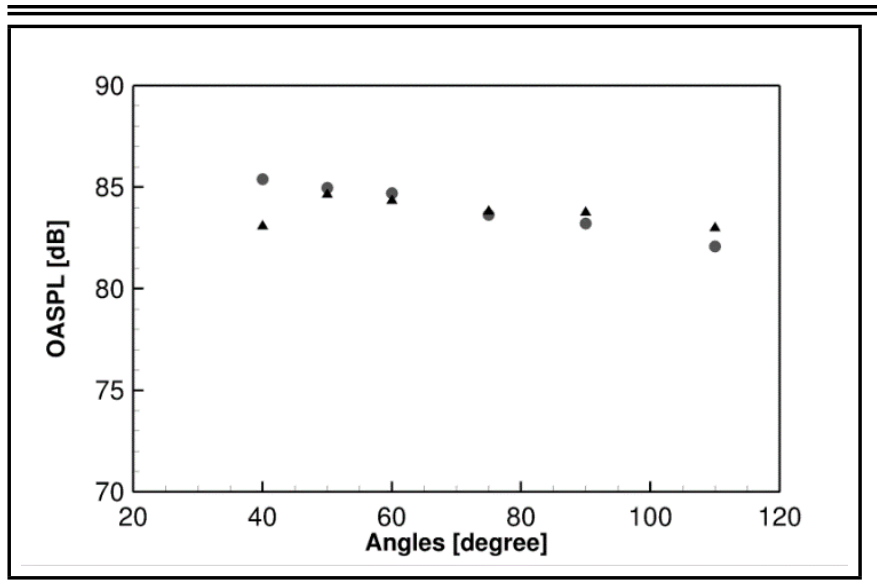

Figure 16. The overall Sound Pressure Level at Mach number 0.25 single jet for angles varying from $40^{\circ}$ up to $110^{\circ}$. $\Delta$ LRT; • Experiments

data, IUTAM/ICA/AIAA-Symposium Gottengen, (1979). http://dx.doi.org/10.1007/978-3-642-81409-9_19

7 Harper-Bourne, M. Jet noise turbulence measurements, Proc. 9th AIAA/CEAS Aeroacoustics Conference and Exhibit, 12-14, (May 2003). http://dx.doi.org/10.2514/6.2003-3214

8 Morris, P. J. and Zaman, K. B. M. Q. Velocity measurements in jets with application to noisesource modeling, Journal of Sound and Vibration, 329, 394-414, (2010). http://dx.doi.org/10.1016/j.jsv.2009.09.024

9 Mielke, A. F., Seasholtz, R. G., Elam, K. A., and Panda, J. Time-average molecular Rayleigh scattering technique for measurement of velocity, density, temperature, and turbulence intensity in high speed nozzle flows, Technical Report AIAA-2004-0706, (2004). http://dx.doi.org/10.2514/6.2004-706

10 Bridges, J., Wernet, M. P., and Brown, C. Control of jet noise through mixing enhancement, Technical Report NASA TM-2003-212335, (2003).

11 Ghahremanian, S. and Moshfegh, B. Numerical and experimental verification of initial, transitional and turbulent regions of free turbulent round jet, Proc. 20th AIAA Computational Fluid Dynamics Conference, Honolulu, Hawaii, 27-30 (2011). http://dx.doi.org/10.2514/6.2011-3697

12 Freund J. B. Noise sources in a low-Reynolds-number turbulent jet at Mach 0.9, J. Fluid Mech., 438, 277-305, (2001). http://dx.doi.org/10.1017/s0022112001004414

13 Stromberg J. L., McLaughlin D. K., and Troutt T. R. Flow field and acoustic properties of a Mach number 0.9 jet at a low Reynolds number, J Sound Vib., 72, 159-76, (1980). http://dx.doi.org/10.1016/0022-460x(80)90650-1

14 Xia, H., Tucker, P. G., and Eastwood, S. Largeeddy simulations of chevron jet flows with noise predictions, Int. J. Heat Fluid Flow, 30, 1067-79, (2009). http://dx.doi.org/10.1016/j.ijheatfluidflow.2009.05.002

15 Birch, S. F., Lyubimov, D. A., and Maslov, V. P. Secundov A. N. Noise prediction chevron nozzle, Proc. 12th AIAA/CEAS Conference 2600, (2006). http://dx.doi.org/10.2514/6.2006-2600
16 Engel, R. C., Silva, C. R. I., and Deschamps, C. J. Application of RANS-based method to predict acoustic noise of chevron nozzles, Applied Acoustics, 79, 153-163, (2014).

17 Silva, C. R. I. Development of a novel RANS-based method for the computational aeroacoustics of high speed jets. PhD Dissertation, So Paulo University, So Paulo, (2011).

18 Pinker, R. A. The enhancement of the QinetiQ noise test facility for larger scale exhaust systems, Technical Report AIAA-2004-3019, (2004). http://dx.doi.org/10.2514/6.2004-3019

19 Proena, A. R. Experimental characterization of velocity and acoustic fields of single-stream subsonic jet, MS diss., Federal University of Uberlndia, Uberlndia, Brazil, (2013). http://dx.doi.org/10.13140/RG.2.2.20281.77928

${ }^{20}$ CFD++ User's manual, Version 8.1. Metacomp Technologies INC.,(2009).

21 Menter, F. R., Zonal two equation k- $\omega$ turbulence models for aerodynamic flows, AIAA Paper 93-2906, (1993). http://dx.doi.org/10.2514/6.1993-2906

22 Pierce, A. D. Acoustics: An Introduction to Its Physical Principles and Applications, McGraw-Hill, New York, (1981).

23 Khavaran, A., Krejsa, E. A., and Kim, C. M. Computation of supersonic jet mixing noise for an axisymmetric cd nozzle using k-w turbulence model, NASA, Technical Report TM 105338, (1992). http://dx.doi.org/10.2514/6.1992-500

24 Jordan, P. and Gervais, Y. Modeling self- and shearnoise mechanisms in inhomogeneous, anisotropic turbulence, Journal of Sound and Vibration, 279, 529-555, (2005). http://dx.doi.org/10.1016/j.jsv.2003.11.045

25 Blokhintzev, D. The propagation of sound in an inhomogeneous and moving medium, The Journal of the Acoustical Society of America, 18 (2), 322-328, (1945). http://dx.doi.org/10.1121/1.1916368

26 Birch, SF, Bukshtab, P. A., Khritov, K. M., Lyubimov D. A., Maslov, V. P., Secundov, A. N., Yakubovsky, K., and $\mathrm{Ya}, \mathrm{A}$. RANS based jet noise prediction procedure, Proc. 13th AIAA/CEAS Conference, 3727, (2007). http://dx.doi.org/10.2514/6.2007-3727

27 Engblom, W. A., Khavaran, A., and Bridges, J. Numerical prediction of chevron nozzle noise reduction using WINDMGBK methodology, Proc. 10th AIAA/CEAS conf., 2979, (2004). http://dx.doi.org/10.2514/6.2004-2979

28 Balsa, T. F., Gliebe, P. R., Kantola, R. A, Wang, J. C. F, and Mani, R. High Velocity Jet Noise Source Location and Reduction: Task 2 - Theoretical Developments and Basic Experiments, Washington-DC: Federal Aviation Administration Report, FAA-RD-76-79-II, 1978. 